University of Windsor

Scholarship at UWindsor

Industrial and Manufacturing Systems

Department of Industrial and Manufacturing

Engineering Publications

Systems Engineering

2003

\title{
A new methodology to evaluate the real options of an investment using binomial trees and Monte Carlo simulations
}

Michele Amico

Zbigniew Pasek

University of Windsor

Farshid Asl

Follow this and additional works at: https://scholar.uwindsor.ca/industrialengpub

Part of the Industrial Engineering Commons

\section{Recommended Citation}

Amico, Michele; Pasek, Zbigniew; and Asl, Farshid. (2003). A new methodology to evaluate the real options of an investment using binomial trees and Monte Carlo simulations. Simulation Conference, 2003. Proceedings of the 2003 Winter, 1, 351-359.

https://scholar.uwindsor.ca/industrialengpub/2

This Conference Proceeding is brought to you for free and open access by the Department of Industrial and Manufacturing Systems Engineering at Scholarship at UWindsor. It has been accepted for inclusion in Industrial and Manufacturing Systems Engineering Publications by an authorized administrator of Scholarship at UWindsor. For more information, please contact scholarship@uwindsor.ca. 


\title{
A NEW METHODOLOGY TO EVALUATE THE REAL OPTIONS OF AN INVESTMENT USING BINOMIAL TREES AND MONTE CARLO SIMULATION
}

\author{
Michele Amico \\ Dipartimento di Tecnologia Meccanica, \\ Produzione ed Ingegneria Gestionale \\ University of Palermo \\ Via delle Scienze, 90128 Palermo, ITALY
}

\author{
Zbigniew J. Pasek \\ Farshid Asl \\ Engineering Research Center for \\ Reconfigurable Manufacturing Systems \\ University of Michigan \\ Ann Arbor, MI 48109-2125, U.S.A.
}

\author{
Giovanni Perrone \\ Dipartimento di Ingegneria e Fisica dell'Ambiente \\ University of Basilicata \\ C.da Macchia Romana, 85100 Potenza, ITALY
}

\begin{abstract}
This paper deals with a new methodology to evaluate the real operating options embedded in a manufacturing system investment. In a single product framework, the demand is assumed as the main source of uncertainty, therefore as a stochastic variable following a Geometric Brownian Motion (GBM). Then, focusing on the real option to expand the capacity at a certain time in the future, we have developed a new approach for the option payoff, looking forward in the time interval from the expansion date to the end of the planning horizon. The payoff function is the expected Net Present Value (NPV), at the expansion date, of the additional investment to increase the capacity, and it is calculated using Monte Carlo simulation. The option value is computed with a binomial tree algorithm. A numerical example and a sensitivity analysis of the option value as a function of some parameters are finally presented.
\end{abstract}

\section{INTRODUCTION}

When dealing with investment decisions, the traditional method is a simple NPV calculation of the different cash flows, in order to select the investment that has the highest positive NPV and discard the projects with negative NPV. In recent years, however, many researchers have shown that conventional economic analysis based on Discounted Cash Flow (DCF) techniques often undervalue projects with real operating options and other strategic interactions
(Miller and Park, 2002). In particular, DCF analysis ignores the "operating flexibility" that gives project managers options to revise decisions in response to changing exogenous economic conditions (Copeland, 2002). The importance of such operating options becomes critical when the environment is highly volatile (extreme variations in market demand and product prices) and the technology is flexible (CNC machines or Reconfigurable Machine Tools - RMTs), thus allowing managerial intervention at a reasonable cost.

Real Option Analysis (ROA), by explicitly capturing the flexibility and its effects on uncertainty, provides for a consistent treatment of risk in the valuation of investment in production systems (Schwarz, 2001). An option is the right, but not the obligation, to take an action in the future. Options are most valuable when there is uncertainty; this is one of the most important shifts in thinking from the real options approach: uncertainty creates opportunities (Amram and Kulatilaka, 1999).

The most common real options are: the waiting-toinvest option, the option to alter the operating scale (option to expand, to contract, to shut down and restart), the option to abandon, and the option to switch (option to change the product mix or processes). For instance, in the valuation of an investment in a Reconfigurable Manufacturing System (RMS), the classical NPV approach might not take correctly into account the value of the real options embedded in the project. Since RMS allow functionality expanding and production capacity upgrading exactly when needed and with a reasonable investment (Koren et al., 1999), the 
value of real options for capacity or mix changes may be critical in the investment decision.

The focus of this paper is the development of an investment evaluation methodology improving the traditional DCF approach when dealing with projects having real operating options in an uncertain world. To accomplish this task, we have developed and tested an advanced decision support tool, able to compute the value of the real options embedded in an investment project.

The paper is organized as follows. Section 2 describes the real option framework, compared to the financial framework and other real option approaches in literature. Section 3 focuses on the particular payoff function developed for this new approach. Section 4 deals with the numerical implementation issues, and Section 5 shows and analyzes the results of a numerical example. Finally, in Section 6 the conclusions and future developments are drawn.

\section{REAL OPTION FRAMEWORK}

The primary objective here is to develop a comparison between the financial option framework and a real option scenario for a production system investment. Let us focus only on the counterpart of the call option, which is the option to expand, i.e. to increase the system capacity by purchase of additional equipment. Let us also assume that the system is producing a single product.

The first step is to find the equivalent of the financial option framework in the production system scenario. This issue has been addressed in various approaches presented in the literature; the most common is the one shown in Table 1 (Trigeorgis, 1991).

Table 1: Financial and Manufacturing Frameworks

\begin{tabular}{c|c}
\hline Financial framework & Manufacturing framework \\
\hline stock price: $S$ & $\begin{array}{c}\text { project DCF without the ini- } \\
\text { tial investment } I_{0}: D C F\end{array}$ \\
\hline stock price volatility: $\sigma$ & volatility of $D C F: \sigma$ \\
\hline exercise time: $T$ & expansion time: $T$ \\
\hline exercise price: $X$ & $\begin{array}{c}\text { investment outlay at time } T \\
\text { to increase the capacity: } I_{1}\end{array}$ \\
\hline risk-free rate: $r$ & risk-free rate: $r$ \\
\hline payoff function: & payoff function: \\
max $\{S(T)-X, 0\}$ & $\max \left\{e \cdot D C F(T)-I_{1}, 0\right\}$ \\
\hline
\end{tabular}

The advantage of this approach is that it even enables use of Black-Scholes model, since it matches perfectly the financial framework: it is enough to substitute the variables. Nevertheless, it also has several weak points and drawbacks:

- the main source of uncertainty is the demand, more than the DCF of the project;

- the payoff of the option, for instance, to expand the capacity, cannot be estimated using only the information at the exercise date, because it depends on the cash flows generated by the new investment in the future, until the end of the planning horizon;

- the exercise price is set a priori and the expansion value is just a percentage $e \cdot 100 \%$ of the asset value at the expansion time, so there is no connection with the capacity change due to the demand increase;

- there is a time delay between the investment time and the date of the additional capacity installation and the system readiness to produce at the new throughput level, and this issue is not considered.

Karsak and Ozogul (2002) value expansion flexibility using American exchange options. Their model has two stochastic variables: the return and the cost of the expansion investment follow two correlated GBM. However, the payoff still depends on the difference between the two assets at the exercise time.

Feinstein and Lander (2002) value real options discounting expected cash flows at a weighted average of the risk-free interest rate and the project cost of capital. It is very close to the traditional NPV approach, but it is limited to one period binomial options.

Our approach differs from the ones presented in literature so far, because its focus is on the demand. Starting from the stochastic process of the demand, a real options framework is built in order to estimate the value of the option to expand the capacity in the future. The focus on a particular real option, for example the option to expand, is not restrictive at all, because all of the following theory can be easily applied to any real option.

The scenario is based on a production system able to manufacture a single product. The initial capacity $C_{0}$ is based on the initial investment $I_{0}$, therefore we are able to produce $C_{0}$ items per year of a single product and to sell them with a contribution margin $m$. We assume that the contribution margin does not change with passing of time.

Primary uncertainty lies in the market demand of the product, which is a stochastic variable $D(t)$. If the demand is $D$ items per year, then we can sell $\min \left\{D, C_{0}\right\}$ items and gain the net income $N I(D)=m \cdot \min \left\{D, C_{0}\right\}$. The previous expression is a very simple net income function, but any more complex function can be used, as long as it is a function of the demand.

Let $f(t, D(t))$ be the pricing function of a real option, depending on the time $t$ and the demand $D(t)$. We assume the demand follows the GBM:

$$
d D(t)=\mu \cdot D(t) \cdot d t+\sigma \cdot D(t) \cdot d W(t)
$$

where $d W(t)$ is a Wiener process, $\mu$ is the expected growth rate (drift) of the demand and $\sigma$ is the volatility of the demand. These two parameters can be easily estimated from historical data. 
The aim is now to find the price $f$ for a derivative depending only on demand and time. The payoff of this derivative at the expiration date $T$ is given by the function $\Phi(D(T))$, which depends on the derivative we are considering and it can be any kind of function. The pricing function is (Bjork, 1998):

$$
f(t, D)=e^{-r \cdot(T-t)} \cdot E_{t, D}^{Q}[\Phi(D(T))] .
$$

The previous expression is the well-known result on pricing a derivative discounting at the risk-free interest rate the expected payoff from the option at its maturity in a risk neutral world. Taking the expectation in a risk neutral world means that we have to use the risk neutral probability measure $Q$. Under this new probability measure, the demand follows a different process, with expected growth rate $\alpha=\mu-\lambda \cdot \sigma$ (risk neutral drift) instead of $\mu$ :

$$
d D=(\mu-\lambda \cdot \sigma) \cdot D \cdot d t+\sigma \cdot D \cdot d W^{Q}
$$

where $d W^{Q}$ denotes a $Q$-Wiener process, and $\lambda$ is the market price of risk, which can be estimated using the Capital Asset Pricing Model (CAPM) (Hull, 2002).

Using the binomial tree approximation with time interval $\Delta t_{1}$, the parameters to be used are:

- up movement coefficient: $u=e^{\sigma \cdot \sqrt{\Delta_{1}}}$,

- down movement coefficient: $d=e^{-\sigma \cdot \sqrt{\Delta_{1}}}=1 / u$,

- risk neutral probability of an up movement: $q=\left(e^{(\mu-\lambda \cdot \sigma) \cdot \Delta t_{1}}-d\right) /(u-d)$.

Obviously, the smaller $\Delta t_{1}$ the better the approximation; we actually choose the number of steps $n_{1}=T / \Delta t_{1}$.

In order to have a stable numeric scheme, the probability $q$ must be limited between 0 and 1 , meaning the following condition must hold:

$$
|\mu / \sigma-\lambda| \cdot \sqrt{\Delta t}<1 \Leftrightarrow n_{1}>T \cdot(\mu-\lambda \cdot \sigma)^{2} / \sigma^{2} .
$$

Therefore, it is always possible to choose a number of steps, $n_{1}$, that is big enough to satisfy the previous constraint.

\section{THE PAYOFF FUNCTION}

Now, we need to define a payoff function for the option value at the expiration date $T$, which is less or equal than the planning horizon $T_{\text {end }}$. At the expiration date, $T$, it is possible to increase the production capacity from $C_{0}$ to $C_{1}$ $\left(C_{1}>C_{0}\right)$ with the investment outlay $I_{1}$.

Hence, the real option we are considering is the counterpart of an European call option with exercise price $I_{1}$. In addition, we assume we know the Net Income of the production, which is a function $N I(D)$ of the demand.

To calculate the payoff we need to look forward to the additional Net Incomes due to the new capacity $C_{1}$ from $T$ to the end of the planning horizon $T_{\text {end }}$, and compare their values, discounted at time $T$, with the additional investment $I_{1}$. So the payoff is basically the net present value of the additional investment at time $T$. This expected NPV might be computed using another binomial tree, starting from each terminal node of the first one. The expected value would be the weighted sum of the NPVs of all the possible paths in the tree, being the weights the probabilities of following each path. To implement this payoff calculation we need to generate and evaluate all the possible paths in the tree. If the time step in the time interval $\left[T, T_{\text {end }}\right]$ is $\Delta t_{2}$ (which can be different than the time step $\Delta t_{1}$ in the time interval $[0, T])$ then there are $n_{2}=\left(T_{\text {end }}-T\right) / \Delta t_{2}$ steps. The number of paths grows exponentially with $n_{2}$, since it is given by $2^{n_{2}}$. Therefore, the execution of this algorithm would be very slow even with small values of $n_{2}$.

To evaluate the payoff function $\Phi(D(T))$, which actually does not depend only on the demand at the exercise date $T$, but on the demand on all the time interval $[T$, $\left.T_{\text {end }}\right]$, we can overcome the drawbacks of the algorithm described in the previous paragraph using the Monte Carlo simulation. Since the payoff is a function of the path in the interval $\left[T, T_{\text {end }}\right]$, and we already know the parameters for a risk neutral simulation of the demand stochastic process, Monte Carlo simulation seems to be the best way to approach this problem.

Let us consider the node $h$ at the end of the binomial tree ( $h$ goes from $-n_{1}$ to $n_{1}$ with step 2 ). The demand in this node is given by $D_{h}(T)=u^{h} \cdot D_{0} \cdot \Delta t_{1}$, where $D_{0}$ is the initial demand. Since in the interval $\left[T, T_{\text {end }}\right]$ we are using a different time step $\Delta t_{1}$, the demand has to be rescaled, so that the initial demand for the simulation starting at time $t_{0}=T$ is finally $D\left(t_{0}\right)=D_{h}(T) \cdot \Delta t_{2} / \Delta t_{1}=u^{h} \cdot D_{0} \cdot \Delta t_{2}$. The capacity must be rescaled as well, so $C_{0,2}=C_{0} \cdot \Delta t_{2}$ and $C_{1,2}=C_{1} \cdot \Delta t_{2}$.

Using the solution to the risk neutral demand process (3), we can easily generate the demand path in the time interval $\left[T, T_{\text {end }}\right]$ :

$$
D\left(t_{j}\right)=D\left(t_{j-1}\right) \cdot \exp \left(\left(\alpha-\frac{\sigma^{2}}{2}\right) \cdot \Delta t_{2}+\sigma \cdot \sqrt{\Delta t_{2}} \cdot \varepsilon_{j}\right)
$$

where $j=1, \ldots n_{2}$ and $\varepsilon_{j} \sim N(0,1)$ are $n_{2}$ independent samples drawn from a standardized normal distribution. At each time $t_{j}$, the income differential due to the increase of capacity is given by:

$$
\Delta N I_{j}=N I\left(D\left(t_{j}\right), C_{0,2}\right)-N I\left(D\left(t_{j}\right), C_{0,1}\right) .
$$


To discount the net incomes at time $T$, the rate to be used is a "risk adjusted rate" of the project, $R$, which is the company cost of capital if the expansion investment has the same risk of the existing business. As a general rule, if the project risk is different from the one of the existing assets, the project should be evaluated at its own opportunity cost of capital. Both the company and the project cost of capital can be estimated using the CAPM (Brealey and Myers, 1996).

Discounting at the rate $R$, the $D C F$ at time $T$ is:

$$
D C F_{i}=\sum_{j=0}^{n_{2}} \exp \left(-R \cdot j \cdot \Delta t_{2}\right) \cdot \Delta N I_{j}
$$

where the subscript $i$ stands for the $i$ th replication. Hence, if we switch the node index from $h$ to $k=0, . . n_{1}$, with $h=$ $2 \cdot k-n_{1}$, then the $i$ th payoff at the node $k$ is:

$$
\Phi_{k, i}=\max \left\{D C F_{i}-I_{1}, 0\right\} .
$$

Denoting with $n$ the number of replications, the expected payoff at the node $k$ is the average over the $n$ replications $\bar{\Phi}_{k}=n^{-1} \cdot \sum_{i=1}^{n} \Phi_{k, i}$.

Once the payoffs at all the terminal nodes are known, the option value $V_{t}$ at time $t<T$, can be computed using the expression (9), where $p_{k}$ is the probability density function of the binomial distribution with parameters $n_{1}$ and $q$, reported in the equation (10):

$$
\begin{gathered}
V_{t}=\exp (-r \cdot(T-t)) \cdot \sum_{k=0}^{n_{1}} p_{k} \cdot \bar{\Phi}_{k} \\
p_{k}=\left(\begin{array}{c}
n_{1} \\
k
\end{array}\right) \cdot q^{k} \cdot(1-q)^{n_{1}-k} .
\end{gathered}
$$

Figure 1 shows the combined methodologies, binomial tree and Monte Carlo simulation.

\section{NUMERICAL IMPLEMENTATION}

Since we are using a discrete time algorithm, to obtain a result that is a good approximation of the real solution (which is unknown), the number of steps in the binomial tree $n_{1}$ should be appropriately high. On the other hand, the number of steps in the simulation equals the number of cash flows to be taken into account in the time interval [ $T$, $\left.T_{\text {end }}\right]$ when comparing the alternative investments "increase the capacity" and "do nothing". Therefore, the time step $\Delta t_{2}$ depends on what time basis the sales are surveyed and the cash flows are computed in the company. We assumed $\Delta t_{2}=0.1$ years.

The code was written and executed with MATLAB ${ }^{\circledR}$. This software tool provides fast implementation, high precision in numerical computations, and quick execution when the code is properly vectorized. In addition, there are tools that allow data import/export with a Microsoft Excel ${ }^{\circledR}$ worksheet. The Monte Carlo simulation was implemented using MATLAB ${ }^{\circledR}$ internal normal random generator; it is a table lookup algorithm developed by Marsaglia, which uses a step function to approximate the normal probability density function ("ziggurat algorithm"). The resulting distribution is exactly normal, the generation is as fast as for the uniformly distributed numbers, and the generator period is $2^{64}$ (Moler, 2001).

As far as the binomial algorithm is concerned, using MATLAB $^{\circledR}$ it is very easy and fast to implement the sum of expression (9), with the statistical function that efficiently returns the binomial probability density function (10), even when $n_{1}$ is a big number.

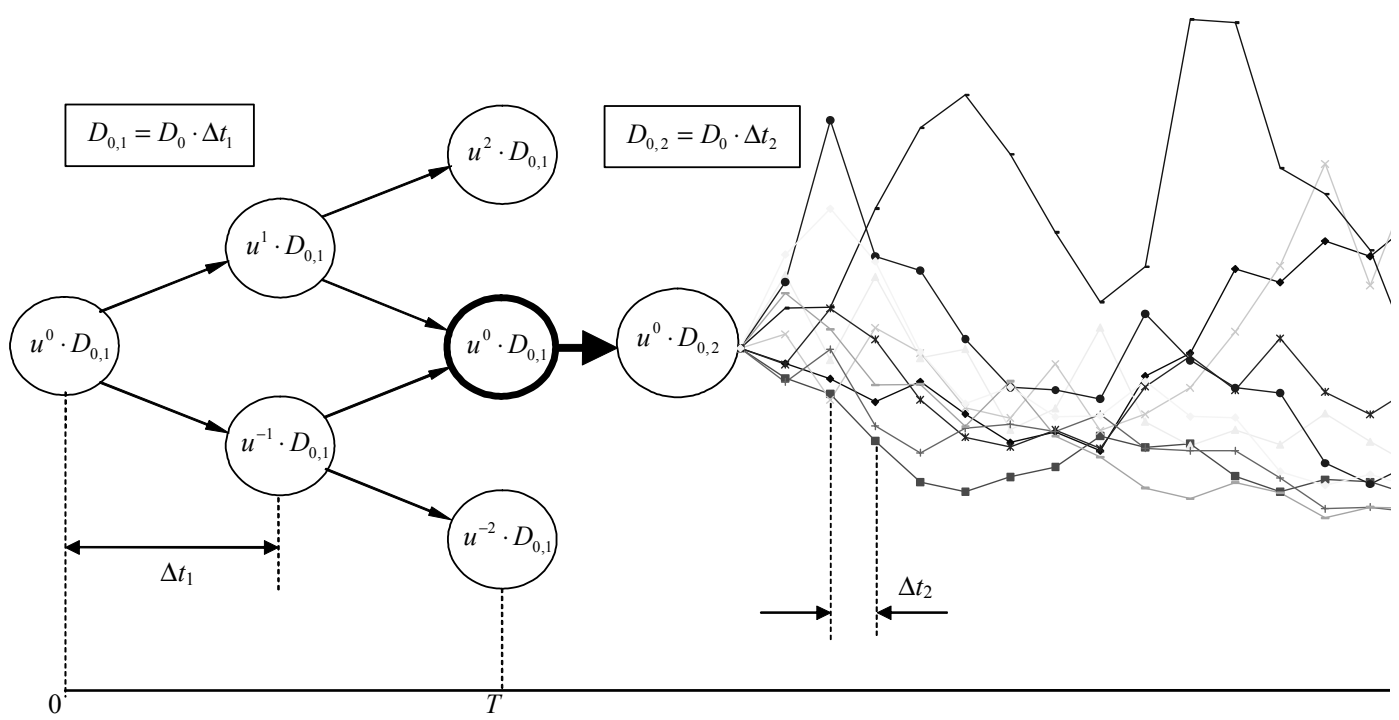

Figure 1: Binomial Tree and Monte Carlo Simulation 
Another critical parameter that deeply affects the goodness of the result is the number of replications $n$, run during the Monte Carlo simulation of the demand paths. Since the number of replications is set to be at least 50 and, above all, the samples $\Phi_{i}$ (in the following we omitted the subscript $k$ because we are considering the payoff for a generic node) are independent, then it is possible to calculate the number of replications in order to control the relative error of the estimate. To perform this task, one should choose $n$ such that the following criterion is met:

$$
\left(z_{1-\alpha / 2} \cdot \sqrt{s^{2} / n}\right) / \bar{\Phi} \leq \gamma /(1-\gamma)
$$

where $z_{1-\alpha / 2}$ is the inverse cumulative standard distribution function, $s^{2}$ is the variance of the samples $\Phi_{i}$, and $\gamma$ is the maximum relative error allowed with probability $1-\alpha$ (Brandimarte, 2001). We assumed $1-\alpha=95 \%$.

The only additional computation required is the estimation of the mean $\bar{\Phi}$ and the variance $s^{2}$ for a first trial of 50 pilot replications; then, solving $n$ from the previous expression, we can find the minimum number of replications to run. After running $n$ replications, the algorithm checks whether the condition (11) still holds with the new estimates of $\bar{\Phi}$ and $s^{2}$; if not, more replications are added solving again the expression (11). Actually, the condition is checked also at some values before $n$, to avoid waiting for useless replications when the initial estimates are not very good. Furthermore, no more replications are added if the condition (11) is not met yet after a running time of 5 minutes since the beginning of that simulation.

In order to reduce the number of replications we implemented the antithetic sampling as a variance reduction technique. Therefore, two demand paths are generated for each replication, using two opposite random sequences $\varepsilon_{j}$ and $-\varepsilon_{j}$. After the payoffs of these sequences are computed, the average of them is the sample $\Phi_{i}$ to be considered. This methodology actually works only if the negative correlation in the input random numbers yields a negative correlation in the output payoffs as well. This actually happens, because of the monotonic relationship between the random input and the payoff, through the composition of the demand function (5), the net income function (6), the $D C F$ function (7) and the payoff function (8). As a result of this variance reduction technique, the number of replications to run in order to get an estimate with a given relative error is, on average, at least $60 \%$ less.

\section{NUMERICAL EXAMPLE}

The numerical data assumed to test the algorithm are reported in Table 2. In the following example we did not consider any historical data, because it is not a real case study. Therefore, the values of the parameters $\mu, \sigma$, and $\lambda$, and all the other input values as well, are just arbitrary numbers.
Table 2: Numerical Data

\begin{tabular}{l|l}
\hline risk free rate & $r=5 \%$ per year \\
planning horizon & $T_{\text {end }}=4$ years \\
exercise time & $T=2$ years \\
initial demand & $D_{0}=125,000$ products per year \\
drift of demand & $\mu=0.9$ per year \\
volatility of demand & $\sigma=0.5$ per year \\
market price of risk & $\lambda=0.8$ per year \\
initial capacity & $C_{0}=160,000$ products per year \\
contribution margin & $m=\$ 15.00$ per product \\
expansion invest- & $I_{1}=\$ 1,000,000$ \\
ment & $C_{1}=250,000$ products per year \\
new capacity & $R=15 \%$ per year \\
risk adjusted rate & \multicolumn{2}{|l}{} \\
\hline
\end{tabular}

We are interested in the option value $V_{0}$ at time $t=0$; hence, everywhere in the following, we will denote it simply as $V$ or "option value."

A first analysis focused on the tuning of the parameters $n_{1}$, number of steps in the binomial tree, and $\gamma$, maximum relative error allowed for the payoffs estimates. They both affect the precision of the option value calculation.

Figure 2 shows the option values when $n_{1}$ increases from 10 to 100 , and $\gamma$ is $10 \%, 5 \%$ and $1 \%$. The precision improvement costs, on average, a running time 3.5 times greater when $\gamma$ decreases from $10 \%$ to $5 \%$. If we want $\gamma$ to decrease from $5 \%$ to $1 \%$, then the running times grow by a factor of 16.5. The convergence when $n_{1}$ increases is almost the same when $\gamma=5 \%$ and $1 \%$; the curve with $\gamma=1 \%$ is just a little smoother. Furthermore, there is no observable improvement tied to $n_{1}$ increase, when the values are already within the precision allowed by $\gamma$. Hence we assumed $n_{1}=50$ and $\gamma=5 \%$. The average running time was about 1.2 minutes. Of course, with better computational power available and higher precision required, these parameters should have greater values.

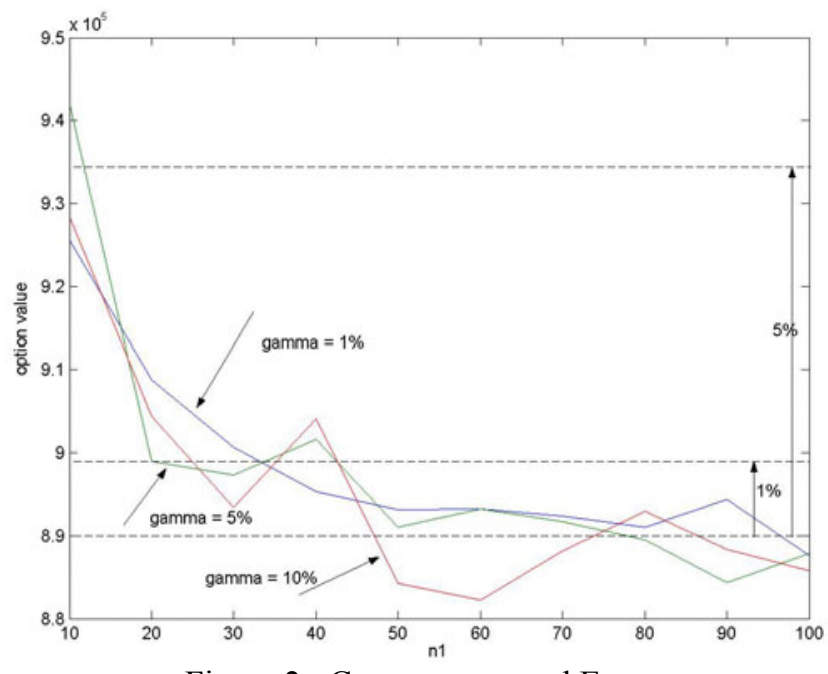

Figure 2: Convergence and Error 
After the algorithm has been tested and its parameters properly tuned, it is very interesting to understand how the option value changes when the main input variables change. The option values have then been computed for different values of the parameters $\mu, \sigma$, and $\lambda$, according to the plan reported in Table 3 , featuring $7 * 7 * 5=245$ points.

Table 3: Experimental Plan $\mu, \sigma, \lambda$

\begin{tabular}{ccccc}
\hline & $\min$ & $\max$ & step & points \\
\hline$\mu$ & -0.75 & 0.75 & 0.25 & 7 \\
$\sigma$ & 0.2 & 1.1 & 0.15 & 7 \\
$\lambda$ & 0 & 2 & 0.5 & 5 \\
\hline
\end{tabular}

Figure 3 shows the contour plot for the function $V(\mu$, $\sigma$ ) when $\lambda=0.5$. When the market price of risk $\lambda$ increases the result is similar, only with much lower option values.
It can be readily noticed in Figure 3, that the option value grows with the increasing demand drift $\mu$, while the dependency on the demand volatility $\sigma$ is more complex.

Looking at the down half of the contour plot, the option value seems to grow with the volatility, exactly like it happens in the financial framework.

However, if we look at the top half of the plot, then the option value decreases when the volatility increases. Furthermore, if we look closer at the area around the half of the plot, the relationship between the option value and the volatility might not even be monotonic.

This result deserves a further analysis, so we have reported the graphs of $V(\mu)$ for the 7 values of $\sigma$, and $V(\sigma)$ for the 7 values of $\mu$. These curves are shown respectively in Figure 4 and Figure 5.

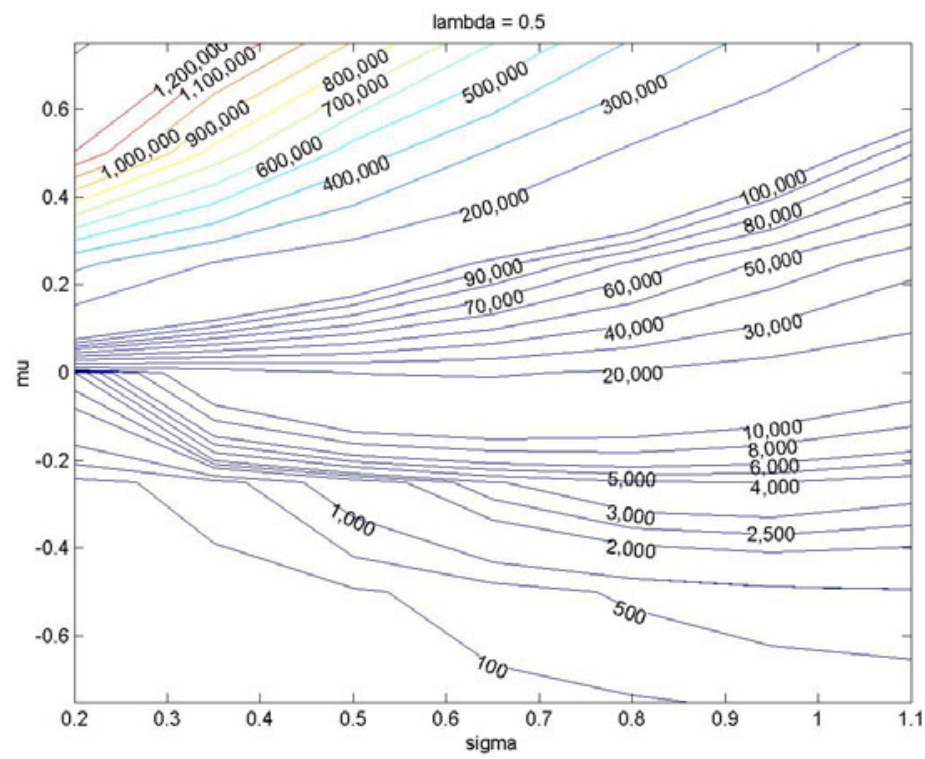

Figure 3: $V(\sigma, \mu)$ when $\lambda=0.5$

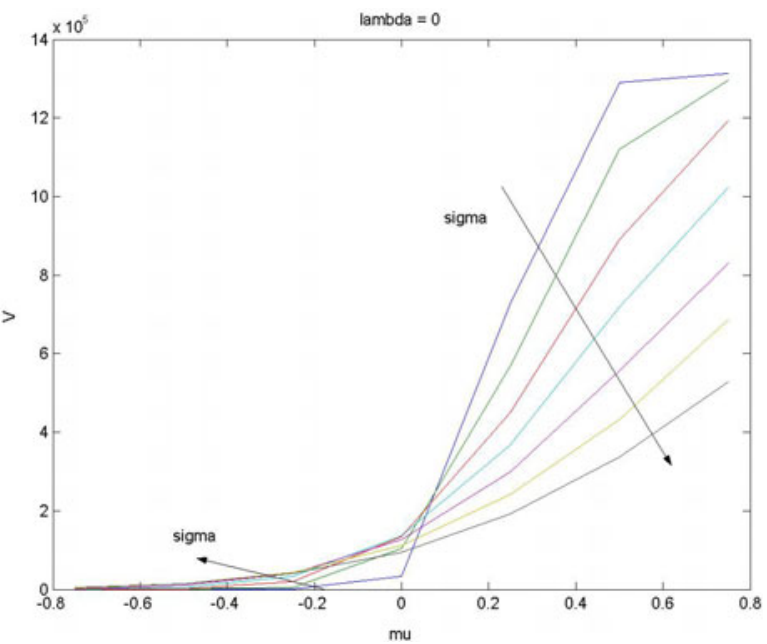

Figure 4: $V(\mu)$ when $\lambda=0$

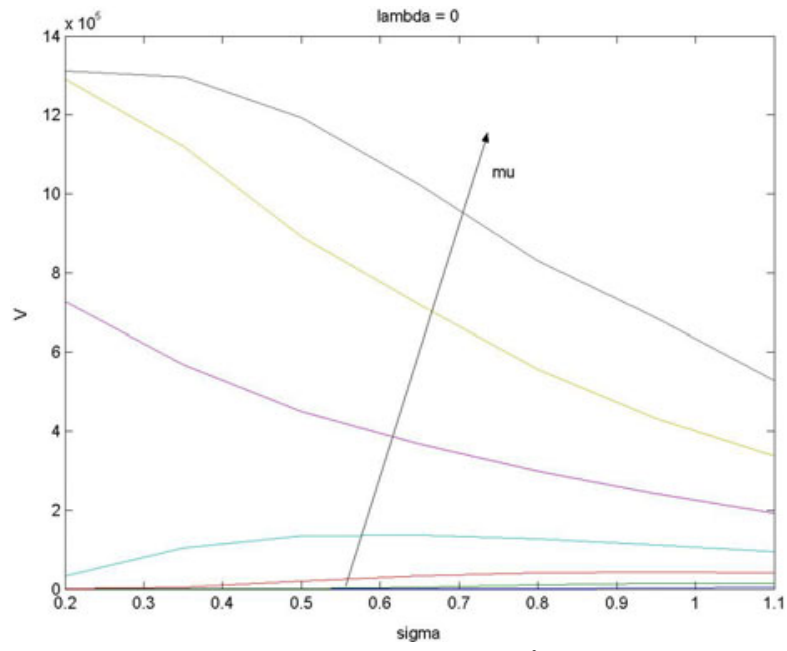

Figure 5: $V(\sigma)$ when $\lambda=0$ 
In Figures 4 and 5 we set $\lambda=0$, primarily because the option values are greater and it improves legibility of the graphs. The option value always increases with respect to the demand drift $\mu$. On the other hand, when $\mu>0$, the option value decreases with the volatility in the range $[0.2$, 1.1]. To better understand what happens when $\mu \leq 0$ we need to zoom in the lower part of Figure 5, because the option values are much smaller when $\mu \leq 0$. This is showed in the following Figure 6.

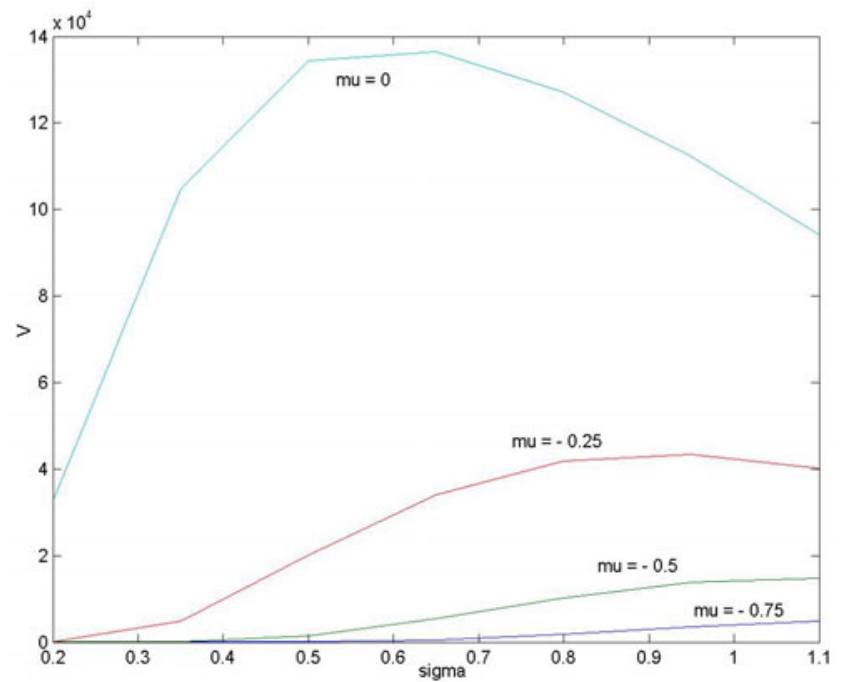

Figure 6: Enlargement of Figure 5 when $\mu \leq 0$

Looking at Figure 6 it is clear that when $\mu=0$ the option value grows up to a maximum, and then it decreases. When $\mu<0$ the maximum shifts to the right, so that within the interval of $\sigma[0.2,1.1]$ the option value looks like it is almost always increasing.

This quite surprising result can be explained looking at the payoff function, which is the main difference between this algorithm for a real European call and the financial European call. In particular, let us consider the demand process (5). The demand changes according to a deterministic term that depends on $\alpha^{\prime}=\alpha-0.5 \sigma^{2}=\mu-\lambda \cdot \sigma-$ $0.5 \sigma^{2}$, and a stochastic term that depends on $\sigma$. When the volatility $\sigma$ increases, $\alpha$ decreases and becomes negative, covering the linear increase and the randomness of the stochastic term. Therefore, the payoff decreases with the volatility, and so does the option value. This effect is highlighted when the drift $\mu>0$, whereas when $\mu<0, \alpha^{\prime}$ is always negative, and the effect of the stochastic term is greater.

Another parameter worthwhile taking into account, is the initial value of the demand $D_{0}$. It is actually better to consider the new parameter $\beta=D_{0} / C_{0}$, which is related to the extra capacity existing in the production system at the beginning (when $\beta<1$ ).
So far we have assumed $\beta=0.78$; now let it go from 0.5 to 1.5 with step 0.25 . Assuming the market price of risk $\lambda=0.5$, the new experimental plan is reported in Table 4 .

Table 4: Experimental Plan $\mu, \sigma, \beta$

\begin{tabular}{ccccc}
\hline & $\min$ & $\max$ & step & points \\
\hline$\mu$ & -0.75 & 0.75 & 0.25 & 7 \\
$\sigma$ & 0.2 & 1.1 & 0.15 & 7 \\
$\beta$ & 0.5 & 1.5 & 0.25 & 5 \\
\hline
\end{tabular}

Figure 7 shows the contour plot for the function $V(\sigma$, $\beta$ ) when $\mu=0.5$. Predictably, the option value grows with $\beta$, whereas it again decreases with the increase of volatility $\sigma$. Like in the previous analysis, this trend reverses when $\mu<0$. Figure 8 shows the same plot when $\mu=-0.5$, and the option value clearly increases with the volatility $\sigma$, at least when $\beta \leq 1$.

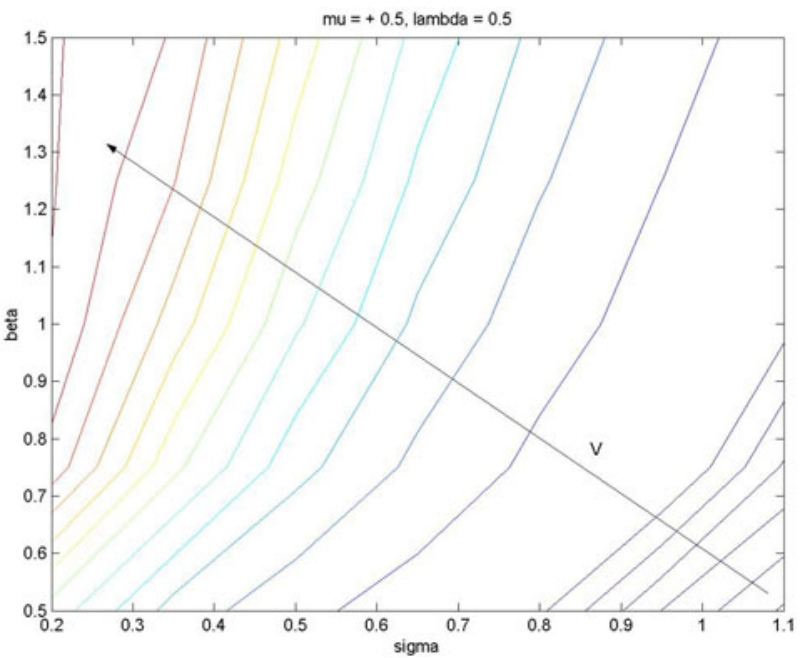

Figure 7: $V(\sigma, \beta)$ when $\mu=0.5$

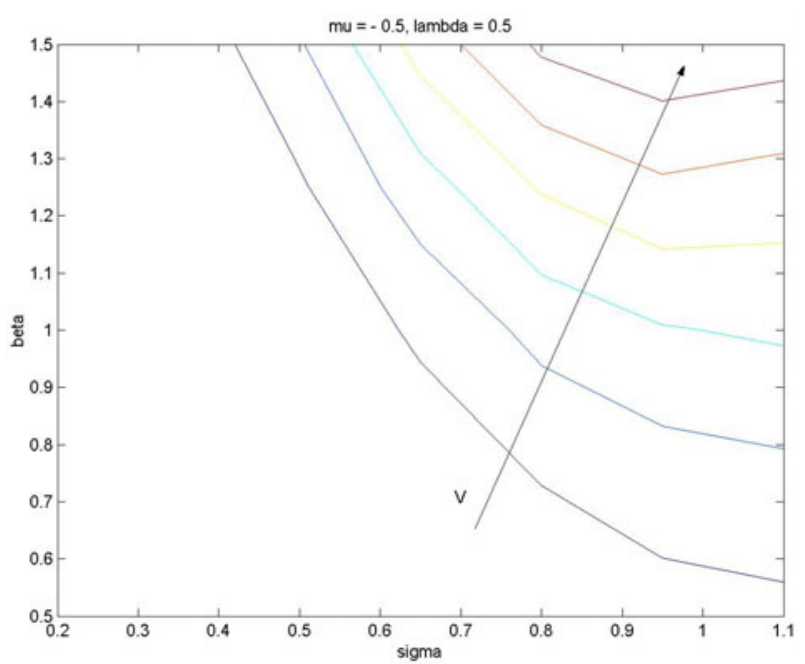

Figure 8: $V(\sigma, \beta)$ when $\mu=-0.5$ 
To better understand the effect of $\beta$, Figure 9 shows the graphs of $V(\sigma)$ for the 5 values of $\beta$. The option value increases with $\sigma$ when $\beta \leq 1$, whereas it has a maximum when $\beta>1$.

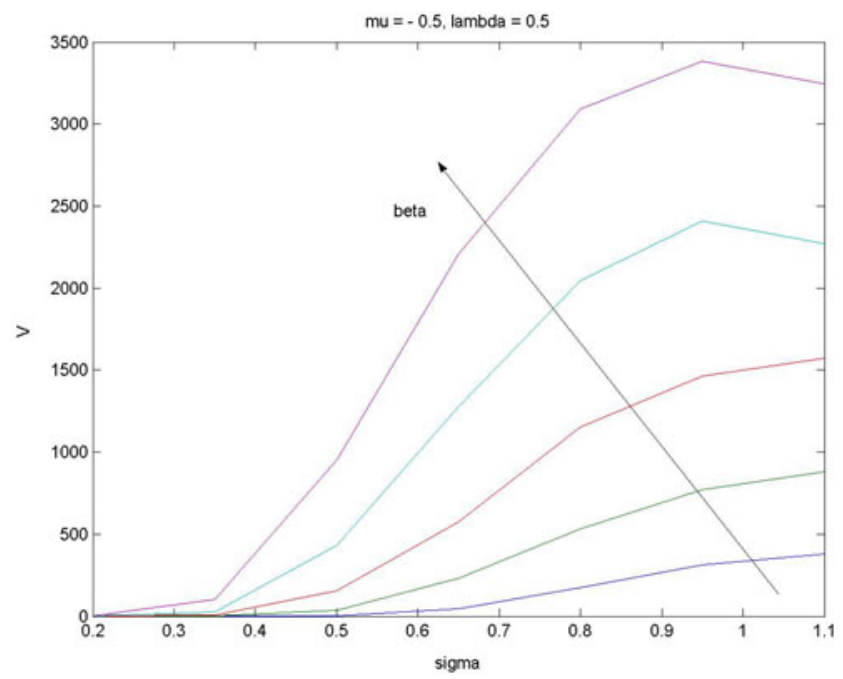

Figure 9: $V(\sigma)$ when $\mu=-0.5$

Like 0 for the drift $\mu, 1$ seems to be a critical value for $\beta$, impacting on the option value as a function of the volatility $\sigma$.

\section{CONCLUSIONS}

In this paper, we have shown a new approach to evaluate real operating options of a production system making a single product. The originality of the methodology is:

- the stochastic variable is the product demand, therefore the market price of risk $\lambda$ has been introduced in the model;

- the payoff function depends on the demand between the expansion date and the end of the planning horizon, and is estimated using Monte Carlo simulation.

These two points are addressing some of the drawbacks of most of the approaches found in the literature, as highlighted in Section 2. The other shortcomings will be addressed in the future developments of this research.

We have focused on an expansion option, and we have developed a tool able to compute the option value for a wide range of input parameters.

A sensitivity analysis has been performed on the option value as a function of the following parameters:

- $\mu$ the drift of the demand,

- $\sigma$ the volatility of the demand,

- $\lambda$ the market price of risk,

- $\beta$ the ratio between initial demand and capacity.

The surprising result is that the option value is not always increasing with the volatility, such as it happens with financial options. We showed that the option value is always increasing with the volatility only when $\mu<0$ and $\beta \leq 1$. When $\mu>0$, it is always decreasing, while for other values it can have a maximum.

Davis (2002) found a very similar result, even though with a different real option model. He shows that growth option value is very likely to be declining in volatility for at- or in-the-money options, whereas it is likely to increase for out-of-the-money growth options. This is not different from our conclusions; actually, when the option value is always increasing with the volatility, the option is almost out-of-the-money, as the reader can notice from the low values on the vertical axes of Figures 6 and 9 (the order of magnitude is $10^{4}$ and $10^{3}$ ). On the other hand, when the option value is always decreasing with the volatility, the option is deeply in-the-money, as the reader can notice from the high values on the vertical axes of Figure 5 (the order of magnitude is $10^{5}$ and $10^{6}$ ).

Davis (2002) states that firms holding at- or in-themoney growth options are justified in avoiding increased market volatility. According to the model presented in this paper, we can extend this result to manufacturing systems holding expansion options.

Nevertheless, the presented results are only the first step in the development of a decision support tool able to evaluate real options embedded in investment in production systems. Future research will focus on different kinds of real options, a varying exercise time (American option), multiple options models, and different kinds of stochastic processes other than the GBM.

\section{ACKNOWLEDGMENTS}

The authors are pleased to acknowledge the financial support of the Engineering Research Center for Reconfigurable Manufacturing Systems (NSF grant \# EEC-9529125), and the assistance from the partners of the ERC (both industry and academia). M. Amico and G. Perrone were supported by the Italian Ministry of University and Research during the course of the presented research.

\section{REFERENCES}

Amram, M. and Kulatilaka, N. 1999. Real options: managing strategic investment in an uncertain world. Harvard Business School Press.

Bjork, T. 1998. Arbitrage Theory in Continuous Time. Oxford University Press.

Brandimarte, P. 2001. Numerical methods in finance: a MATLAB-based introduction. John Wiley \& Sons.

Brealey, R. A. and Myers, S. C. 1996. Principles of Corporate Finance. McGraw-Hill.

Copeland, T. 2002. The Real-Options Approach to Capital Allocation. IEEE Engineering Management Review, First Quarter 2002: 82-85. 
Davis, G. D. 2002. The Impact of Volatility on Firms Holding Growth Options. The Engineering Economist 47: 213-231.

Feinstein S. P. and Lander D. M. 2002. A Better Understanding of Why NPV Undervalues Managerial Flexibility. The Engineering Economist 47: 418-435.

Hull, J. C. 2002. Options, Futures and other Derivatives. Prentice Hall.

Karsak, E. E. and Ozogul, C. O. 2002. An Options Approach to Valuing Expansion Flexibility in Flexible Manufacturing System Investments. The Engineering Economist 47: 169-193.

Koren, Y., Heisel, U., Jovane, F., Moriwaki, T., Pritschow, G., Ulsoy, G., and Van Brussel, H. 1999. Reconfigurable Manufacturing Systems. Annals of the CIRP 48/2, Keynote Paper.

Miller, T.L. and Park C.S. 2002. Decision Making Under Uncertainty-Real Options to the Rescue? The Engineering Economist 47: 105-150.

Moler C. 2001. Ziggurat algorithm generates normally distributed random numbers. MATLAB ${ }^{\circledR}$ News \& Notes Spring 2001. Available online via $<$ http: //www . mathworks.com/company/newsletter/cle vescorner/spring01_cleve.shtml> [accessed March 2003].

Schwarz, E. S. 2001. Real options and investment under uncertainty: classical readings and recent contributions, edited by Eduardo S. Schwarz and Lenos Trigeorgis, MIT Press.

Trigeorgis, L. 1991. A Log-Transformed Binomial Numerical Analysis Method for Valuing Complex MultiOption Investments. Journal of Financial and Quantitative Analysis 26: 309-326.

\section{AUTHOR BIOGRAPHIES}

MICHELE AMICO is a Ph.D. student in Production Engineering at the Department of Mechanical Technology, Production and Industrial Engineering at the University of Palermo, Italy. He has a Master Degree in Industrial Engineering. His research mainly focused on the development of a Decision Support System for capacity planning decision in advanced manufacturing systems, the shop floor control with multi agents systems, the study of real time methods for error handling in Reconfigurable Manufacturing Systems. After one year as a visiting scholar at the ERC/RMS of the University of Michigan, Ann Arbor, he started working on the applications of real options theory to the evaluation of investments in production systems. His e-mail address is <amico@dtpm.unipa.it>.

ZBIGNIEW J. PASEK is the operations manager and assistant research scientist for the NSF Engineering Research Center for Reconfigurable Manufacturing Systems at the College of Engineering, University of Michigan. His re- search interests include risk management, open architecture control for reconfigurable systems, e-diagnostics for maintenance, and software development for informal technology education. He is a member of IEEE, ASME, and SME. He can be reached at<zbigniew@umich.edu> .

FARSHID MAGHAMI ASL has completed his Ph.D. in Mechanical Engineering and his Masters in Financial Engineering in 2002 while working in the NSF Engineering Research Center (ERC) for Reconfigurable Manufacturing Systems in the University of Michigan. Before joining the University of Michigan, he studied in Villanova University for his M.S., and KNT University in Tehran for his B.S. degrees in Mechanical Engineering. His current research includes: Stochastic Optimal Investment in Capacity, Real Options and Risk Management in Manufacturing Operations. He can be reached at $<$ fasl@umich. edu $>$.

GIOVANNI PERRONE is a Full Professor in the area of Manufacturing and Production Systems and he teaches Manufacturing, Computer Integrated Manufacturing and Industrial Engineering at the Faculty of Engineering of the University of Basilicata in Potenza, Italy. He also teaches Manufacturing at the University of Palermo, Italy. His principal interests are in Production Engineering and Management, Operations Management, Advanced and Intelligent Manufacturing Systems, Production Economics and Soft Computing Techniques. He is a member of IIE, IEEE, corresponding member of CIRP and member of AITEM (Associazione Italiana di Tecnologia Meccanica - Italian association for manufacturing). He acts as reviewer for the following journals: International Journal of Production Research, International Journal of Production Economics and the Engineering Economist. He can be contacted by e-mail at $<$ perrone@unibas.it>. 